\title{
Apical membrane expression of distinct sulfated glycans represents a novel marker of cholangiolocellular carcinoma
}

\author{
Hitomi Hoshino ${ }^{1}$, Makoto Ohta ${ }^{2}$, Makoto Ito ${ }^{3}$, Kenji Uchimura ${ }^{4}$, Yasuhiro Sakai ${ }^{5}$, Takeshi Uehara ${ }^{6}$, Shulin Low ${ }^{1}$, \\ Mana Fukushima ${ }^{5}$ and Motohiro Kobayashi ${ }^{1}$
}

Intrahepatic cholangiocarcinoma (ICC) is the second most common primary liver neoplasm, followed by hepatocellular carcinoma. ICC can be further subclassified as (i) perihilar and (ii) peripheral types, the latter histologically resembling small-sized intrahepatic bile ducts, such as interlobular bile ducts, cholangioles/ductules and the canals of Hering. Cholangiolocellular carcinoma (CoCC), now classified by the World Health Organization as a subtype of combined hepatocellular-cholangiocarcinoma, is currently regarded as a subtype of peripheral-type ICC. The present study was undertaken to determine whether sulfated glycans recognized by the MECA-79 monoclonal antibody could serve as a CoCC marker. Using immunohistochemistry, we show that MECA-79 sulfated glycans are preferentially expressed at the apical membrane of cholangiocytes found in small-sized intrahepatic bile ducts in normal liver and in canalicular structures formed in CoCC. We also report that apical membrane MECA-79 sulfated glycan expression colocalizes with that of mucin 1 (MUC1) core proteins. We also present immunoblotting of Chinese hamster ovary cells overexpressing FLAGtagged MUC1 to show that MUC1 serves as a MECA-79 scaffold. Furthermore, we report that SSP-25 human ICC cells overexpressing $\mathrm{N}$-acetylglucosamine-6-O-sulfotransferase 2 (GlcNAc6ST-2), but not GlcNAc6ST-1, exhibit membrane expression of MECA-79 sulfated glycans, suggesting that GlcNAc6ST-2 catalyzes MECA-79 epitope biosynthesis in cholangiocytes. Moreover, both wild-type and GlcNAc6ST-1 knockout mice exhibit apical membrane MECA-79 expression in small-sized intrahepatic bile ducts, namely interlobular bile ducts, whereas MECA-79 expression was completely absent in comparable tissues from GlcNAc6ST-1 and GlcNAc6ST-2 double knockout mice. These data collectively indicate that apical membrane localization of MUC1 proteins decorated with GlcNAc6ST-2-dependent MECA-79 sulfated glycans may mark cholangiocytes with cholangiolar/ductular differentiation and could serve as a useful CoCC marker.

Laboratory Investigation (2016) 96, 1246-1255; doi:10.1038/labinvest.2016.104; published online 17 October 2016

Intrahepatic cholangiocarcinoma (ICC) is the second most common primary liver neoplasm, followed by hepatocellular carcinoma (HCC). ${ }^{1,2}$ ICC can be further subclassified into (i) perihilar type (also called large bile duct type) and (ii) peripheral type (also called small bile duct type or cholangiolar type), ${ }^{2-6}$ and both subtypes are thought to arise from topologically different intrahepatic bile ducts. ${ }^{5}$ As named, perihilar type emerges from perihilar large-sized intrahepatic bile ducts, such as segmental and septal bile ducts, which are lined with mucous-producing tall cylindrical cholangiocytes (biliary epithelial cells), ${ }^{7}$ whereas peripheral type originates in peripheral small-sized intrahepatic bile ducts, such as interlobular bile ducts, cholangioles/ductules, and the canals of Hering, ${ }^{8}$ which are lined with non-mucousproducing cuboidal cholangiocytes. ${ }^{1,2}$

Cholangiolocellular carcinoma (CoCC) (also called bile ductular carcinoma $\mathrm{a}^{3}$ ), classified as cholangiolocellular type of combined hepatocellular-cholangiocarcinoma with stem cell features in the latest (4th) edition of the World Health Organization (WHO) classification, ${ }^{9}$ was first described by Steiner and Higginson in $1959 .{ }^{10}$ For over a half century since then, CoCC has been categorized as a special subtype of

\footnotetext{
'Department of Tumor Pathology, Faculty of Medical Sciences, University of Fukui, Eiheiji, Japan; ${ }^{2}$ Department of Pathology, Fukui Red Cross Hospital, Fukui, Japan; ${ }^{3}$ Department of Pathology and Laboratory Medicine, Kariya Toyota General Hospital, Kariya, Japan; ${ }^{4}$ Department of Biochemistry, Nagoya University Graduate School of Medicine, Nagoya, Japan; ${ }^{5}$ Department of Molecular Pathology, Shinshu University Graduate School of Medicine, Matsumoto, Japan and ${ }^{6}$ Department of Laboratory Medicine, Shinshu University School of Medicine, Matsumoto, Japan

Correspondence: Professor M Kobayashi, MD, PhD, Department of Tumor Pathology, Faculty of Medical Sciences, University of Fukui, 23-3 Matsuoka-Shimoaizuki, Eiheiji, Fukui 910-1193, Japan.

E-mail: motokoba@u-fukui.ac.jp

Received 8 March 2016; revised 25 August 2016; accepted 30 August 2016
} 
peripheral-type ICC. ${ }^{5,6,11}$ Histologically, CoCC is described as well-differentiated adenocarcinoma forming anastomosed antler-like glands with edematous fine fibrous stroma, resembling a ductular reaction seen in several acute and chronic liver diseases, including massive hepatic necrosis. ${ }^{10-12}$ CoCC is thought to originate in the peripherally distributed biliary ductular system, including cholangioles/ductules and the canals of Hering, ${ }^{8}$ where hepatic progenitor cells (HPCs) are located. ${ }^{5,11,12}$ As HPCs can differentiate into either hepatocytes or cholangiocytes, ${ }^{11}$ they are thought to be the origin of combined hepatocellular-cholangiocarcinoma. However, the histogenesis of CoCC and combined hepatocellular-cholangiocarcinoma remains controversial, and definite conclusions relevant to diagnostic criteria for these diseases have not yet been reached.

Several molecules have been proposed as CoCC markers, including cytokeratin 19, ${ }^{12,13}$ neural cell adhesion molecule $1,{ }^{14} \mathrm{~N}$-cadherin, ${ }^{1}$ and mucin 1 (MUC1). ${ }^{7,15,16}$ Among them, MUC1, also known as epithelial membrane antigen (EMA), is generally thought to be most useful to distinguish CoCC from perihilar-type ICC: CoCC shows an apical membrane MUC1 staining pattern, whereas MUC1 staining is diffuse and cytoplasmic in perihilar-type ICC. $7,15,16$

Transmembrane MUC1 protein is present in the apical membrane of glandular epithelial cells in numerous organs and tissues, where it functions primarily in lubrication and cell protection. ${ }^{17,18}$ MUC1 is translated as a single polypeptide chain that undergoes autoproteolytic cleavage to form two subunits that interact non-covalently. ${ }^{19}$ The large $\mathrm{N}$-terminal subunit consists of a long extracellular domain, and the small C-terminal subunit contains a short extracellular domain, a transmembrane region and a cytoplasmic tail. ${ }^{20}$ The larger subunit harbors a serine/threonine-rich region with a highly polymorphic sequence motif termed the variable number of tandem repeats (VNTR), which is often extensively $O$ glycosylated with a battery of Lewis blood group-related carbohydrate antigens, such as sialyl Lewis A (also known as CA19-9) and sialyl Lewis X. ${ }^{17,21,22}$

A sulfated form of sialyl Lewis X, specifically 6-sulfo sialyl Lewis $\mathrm{X}$, is displayed on high endothelial venules (HEVs) in secondary lymphoid organs such as lymph nodes, tonsils and Peyer's patches, and functions in physiological recirculation of lymphocytes from the bloodstream into lymphoid organs, a process termed lymphocyte 'homing. ${ }^{23}$ Although absent under normal conditions, 6-sulfo sialyl Lewis $\mathrm{X}$ is also displayed on HEV-like vessels induced in chronic inflammatory gastrointestinal diseases such as chronic Helicobacter pylori gastritis ${ }^{24,25}$ and ulcerative colitis, ${ }^{26,27}$ where it functions in pathologic lymphocyte recruitment. Since the discovery that 6-sulfo sialyl Lewis X-capped glycans on HEVs function as ligands of L-selectin on lymphocytes, ${ }^{28-30}$ investigators have detected those glycans using the monoclonal antibody MECA-79, ${ }^{31}$ whose epitope is 6-sulfo $\mathrm{N}$-acetyllactosamine attached to extended core $1 O$-glycans, Gal $\beta 1 \rightarrow 4$ $\left(\right.$ sulfo $\rightarrow 6$ ) GlcNAc $\beta 1 \rightarrow 3 \mathrm{Gal} \beta 1 \rightarrow 3 \mathrm{GalNAc} \alpha 1 \rightarrow$ Ser/Thr. ${ }^{32}$
Previously, we demonstrated that HEV-like vessels induced in type 1 autoimmune pancreatitis were positively immunostained by MECA-79 monoclonal antibody. ${ }^{33}$ In that study, we unexpectedly discovered a sizable fraction of interlobular pancreatic ducts that were also MECA-79 positive. ${ }^{33}$ Thus, we concluded that the biliary system, which is connected to the pancreatic duct system to form the pancreaticobiliary system, ${ }^{7}$ is also decorated with MECA-79 sulfated glycans. It remained unknown whether MUC1 was the scaffold protein for those glycans.

In the present study, we demonstrate that MECA-79 sulfated glycans are preferentially expressed in apical membranes of small-sized intrahepatic bile ducts as well as on canalicular structures in CoCC. Moreover, apical membrane MECA-79 positivity colocalized with MUC1 core proteins. These findings suggest that apical membrane localization of MUC1 protein decorated with MECA-79 sulfated glycans may be a distinct feature of cholangiocytes with cholangiolar/ductular differentiation and serve as a CoCC marker.

\section{MATERIALS AND METHODS Human Tissue Samples}

In the present study, the diagnosis of CoCC was made when cases of peripheral-type ICC (in a broad sense) fulfilled all four criteria as follows: (i) well-differentiated adenocarcinoma forming small monotonous and/or anastomosing glands, (ii) which are composed of cuboidal non-mucousproducing neoplastic cholangiocytes, (iii) with fine fibrous stroma, and (iv) luminal surface of canalicular structures were positively immunostained by MUC1. Formalin-fixed, paraffin-embedded tissue blocks of CoCC liver tissue $(n=20 ; 6$ males and 14 females; age range, 48-85 years; median, 71 years) were retrieved from the pathological archives of Shinshu University Hospital and its affiliated hospitals. Liver tissue blocks with peripheral-type ICC ( $n=26$; 15 males and 11 females; age range, $28-85$ years; median, 70 years), perihilar-type ICC $(n=25 ; 18$ males and 7 females; age range, $54-85$ years; median, 65 years) and HCC $(n=25 ; 24$ males and 1 female; age range, $44-85$ years; median, 68 years) were also retrieved as controls. Detailed clinicopathological information, including background liver diseases, of these patients is listed in Supplementary Table 1. Analysis of human liver tissues was approved by the Ethics Committee of Shinshu University School of Medicine and by the Ethics Committee of Faculty of Medical Sciences, University of Fukui.

\section{Mouse Tissue Samples}

Mice deficient in either $\mathrm{N}$-acetylglucosamine-6-O-sulfotransferase 1 (GlcNAc6ST- $)^{34}$ or both GlcNAc6ST-1 and GlcNAc6ST-2 were generated as described. ${ }^{35}$ Mutant mice and wild-type littermates were maintained in the animal facility of Nagoya University Graduate School of Medicine in accordance with institutional guidelines. For tissue analysis, 
after deep anesthesia with sodium pentobarbital, mice were perfused with phosphate-buffered saline (PBS), and their livers were removed, fixed in neutralized $10 \%$ formalin at $4{ }^{\circ} \mathrm{C}$ for $24 \mathrm{~h}$, and embedded in paraffin. The experimental protocol was approved by the Animal Research Committee of Nagoya University.

\section{Immunohistochemistry}

The following monoclonal antibodies served as primary antibodies: GP1.4, recognizing the sequence PDTRPAPGS in the VNTR of human MUC1 (mouse IgG; Leica Biosystems, Nussloch, Germany $)^{36}$ and MECA-79, recognizing 6-sulfo $N$ acetyllactosamine attached to extended core $1 O$-glycans (rat IgM; BD Biosciences, San Jose, CA). ${ }^{31,32}$ MUC1 immunohistochemistry was undertaken using the Histofine system (Nichirei, Tokyo, Japan) according to the manufacturer's protocol, and that for MECA-79 was carried out using an indirect method as described. ${ }^{37}$ MUC1/MECA-79 double immunofluorescence was carried out as described. ${ }^{38}$

\section{Cell Culture}

The human ICC cell line SSP-25 (Riken Bioresource Center, Tsukuba, Japan) was cultured in RPMI-1640 medium (Thermo Fisher Scientific, Waltham, MA, USA) supplemented with 10\% fetal bovine serum (HyClone, South Logan, UT) at $37^{\circ} \mathrm{C}$ in a humidified atmosphere of $5 \% \mathrm{CO}_{2}$. Chinese hamster ovary $(\mathrm{CHO})$ cells stably expressing $\mathrm{CD} 34$ decorated with 6-sulfo sialyl Lewis $\mathrm{X}$ attached to extended core $1 \mathrm{O}$ glycans, sialic acid $\alpha 2 \rightarrow 3 \mathrm{Gal} \beta 1 \rightarrow 4[\mathrm{Fuc} \alpha 1 \rightarrow 3$ (sulfo $\rightarrow 6$ )] GlcNAc $\beta 1 \rightarrow 3 \mathrm{Gal} \beta 1 \rightarrow 3 \mathrm{GalNAc} \alpha 1 \rightarrow \operatorname{Ser} / \mathrm{Thr} \quad$ (termed $\mathrm{CHO} / \mathrm{CD} 34 / \mathrm{F} 7 / \mathrm{C} 1 / \mathrm{LSST}$ cells), were established and cultured as described. ${ }^{39}$

\section{RT-PCR}

Total RNA was extracted and single-stranded cDNA synthesized as described. ${ }^{38}$ PCR was performed also as described. ${ }^{38}$

\section{Expression of GlcNAc6STs in SSP-25 Cells}

SSP-25 cells were transiently transfected with pcDNA1.1GlcNAc6ST-1, ${ }^{27,40,41}$ pcDNA1.1-GlcNAc6ST-2, ${ }^{24,42}$ or pcDNA1.1 (mock) using Lipofectamine Plus (Thermo Fisher Scientific) according to the manufacturer's instructions. Cells were analyzed $36 \mathrm{~h}$ later. Expression of GlcNAc6ST-1 protein in GlcNAc6ST-1-transfected cells was confirmed by immunofluorescence with an anti-GlcNAc6ST-1-N antibody as described. ${ }^{41}$

Construction of a FLAG-Tagged MUC1 Expression Vector cDNA encoding FLAG-tagged MUC1 was constructed as described previously ${ }^{17}$ using an overlapping PCR method described elsewhere. ${ }^{43,44}$ Briefly, with outer primers and internal complementary primers harboring the FLAG tag sequence, two overlapping PCR fragments were amplified in the first round of PCR using the cDNA clone AK301575, which encodes a human MUC1 splicing variant (Biological
Resource Center, National Institute of Technology and Evaluation, Kisarazu, Japan) as a template. In the second round of PCR, the full-length MUC1 coding region with a FLAG epitope tag was generated using only outer primers and first-round PCR products as a template. Primers used were as follows: 5'-TCAAgCTTCACCACCACCATgACACCgggCAC -3' (outer forward primer), 5'-TgCTCgAgCTACAAgTTggCA gAAgTggCTgC-3' (outer reverse primer), 5'-ACTgAgAA gAATgCTgACTACAAAgACgATgACgACAAggTgAgTATgAC CAgC-3' (inner forward primer), and its complementary inner reverse primer 5'-gCTggTCATACTCACCTTgTCgTCA 'TCgTCTTTgTAgTCAgCATTCTTCTCAgT-3' (HindIII and XhoI sites underlined; FLAG epitope in bold). PCR products were inserted into pCR-Blunt (Thermo Fisher Scientific), resulting in pCR-Blunt-MUC1/FLAG, and the sequence confirmed. pCR-Blunt-MUC1/FLAG was digested with HindIII and XhoI, and the insert ligated into pcDNA3.1/ Hygro HindIII and XhoI sites, resulting in pcDNA3.1/HygroMUC1/FLAG.

\section{Immunoprecipitation and Western Blot Analysis}

$\mathrm{CHO} / \mathrm{CD} 34 / \mathrm{F} 7 / \mathrm{C} 1 / \mathrm{LSST}$ cells were transiently transfected with pcDNA3.1/Hygro-MUC1/FLAG or pcDNA3.1/Hygro (mock) using Lipofectamine Plus. After $36 \mathrm{~h}$, cells were lysed with a sonicator in $1 \%$ sodium dodecyl sulfate (SDS) supplemented with a protease inhibitor cocktail (Roche, Basel, Switzerland). Cell lysates were then incubated with anti-FLAG M2 monoclonal antibody (mouse IgG; SigmaAldrich, St Louis, MO) at $4{ }^{\circ} \mathrm{C}$ for $60 \mathrm{~min}$ on a rotator. Mouse IgG (Beckman Coulter, Indianapolis, IN) served as a negative control. Protein G Sepharose Fast Flow (Sigma-Aldrich) was prepared according to the manufacturer's instructions and added to the cell lysate/antibody mix, and then incubated at $4{ }^{\circ} \mathrm{C}$ for $60 \mathrm{~min}$ on a rotator. Beads were washed in PBS five times and lysed in a sample buffer, and then Western blot analysis was carried out as described. ${ }^{45}$ Briefly, after incubation at $65^{\circ} \mathrm{C}$ for $15 \mathrm{~min}$, samples were subjected to $5 \%$ SDS-polyacrylamide gel electrophoresis (SDS-PAGE) and transferred onto a polyvinylidene difluoride membrane (Millipore, Billerica, MA). After blocking in Tris-buffered saline containing $5 \%$ skim milk for $60 \mathrm{~min}$, the membrane was incubated with anti-FLAG or MECA-79 monoclonal antibodies at $4{ }^{\circ} \mathrm{C}$ overnight, followed by host- and isotypematched horseradish peroxidase-conjugated secondary antibodies (Jackson ImmunoResearch, West Grove, PA). The membrane was developed using SuperSignal West Dura Extended Duration Substrate (Thermo Fisher Scientific) and evaluated using a luminescent image analyzer LAS-4000 (GE Healthcare, Little Chalfont, UK).

\section{Statistical Analysis}

Differences between groups were statistically analyzed by Fisher's exact test using the GraphPad Prism 5 software (GraphPad Software, La Jolla, CA). $P$-values $<0.05$ were considered significant. 


\section{RESULTS}

\section{Expression Patterns of MECA-79 Sulfated Glycans in} Intrahepatic Bile Ducts

To determine whether cholangiocytes that constitute intrahepatic bile ducts express MECA-79 sulfated glycans, we first carried out immunohistochemistry of normal liver tissue sections using MECA-79 monoclonal antibody. As shown in Figure 1 (upper panels), small-sized intrahepatic bile ducts, such as interlobular bile ducts (arrows), cholangioles/ ductules, and the canals of Hering (arrowheads), were preferentially immunostained for MECA-79, chiefly in an apical membrane pattern. However, when we examined largesized intrahepatic bile ducts (Figure 1, lower panels), the largest ones, such as segmental bile ducts (arrows), were MECA-79 negative, and the smaller the ducts (arrowheads), the more MECA-79 positivity they exhibited, although staining was diffuse and cytoplasmic. Hepatocytes were completely negative for MECA-79 sulfated glycans (see Figure 1, upper middle panel). This staining pattern was observed in all normal liver regions in all samples examined. Interestingly, expression patterns of MUC1 core proteins (Figure 1, right column) were comparable to those of MECA-79 sulfated glycans (Figure 1, middle column); however, MUC1 core proteins were expressed weakly in the cytoplasm of cholangiocytes that constitute large-sized intrahepatic bile ducts (Figure 1, lower right panel). Moreover, in small-sized intrahepatic bile ducts, MUC1 core protein expression was restricted to interlobular bile ducts, and no signals were detected in cholangioles/ductules and the canals of Hering (Figure 1, upper right panel). These findings indicate that MECA-79 immunohistochemistry is useful to identify small-sized intrahepatic bile ducts and, compared with MUC1 (EMA) staining, can identify even the smallest bile ducts, namely the canals of Hering.

\section{Apical Membrane Expression of MECA-79 Sulfated Glycans in CoCC}

We then determined whether CoCC tissues, which histologically resemble small-sized intrahepatic bile ducts, express MECA-79 sulfated glycans. As shown in Figure 2 (top middle panel), MECA-79 sulfated glycans were preferentially expressed along the luminal surface of canalicular structures formed in CoCC in 18 of $20(90.0 \%)$ cases examined. A similar MECA-79 staining pattern was observed in 12 of 26 (46.2\%) peripheral-type ICC cases (Figure 2, second row, middle panel). By contrast, in perihilar-type ICC $(n=25)$, apical membrane expression of MECA-79 sulfated glycans was not observed and only a small fraction of carcinoma cells weakly expressed these sulfated glycans in the cytoplasm (Figure 2, third row, middle panel). HCC tissues $(n=25)$ were completely negative for MECA-79 sulfated glycans (Figure 2, bottom middle panel). Table 1 summarizes the incidence of apical membrane expression of MECA-79 sulfated glycans in all primary liver tumors examined
( $n=96$ in total). The incidence of MECA-79 apical membrane expression among CoCC cases was greater than that among HCC $(P<0.0001)$, perihilar-type ICC $(P<0.0001)$, or peripheral-type ICC $(P=0.0022)$ cases with high statistical significance. Intriguingly, the expression pattern of MUC1 core proteins in CoCC resembled that of MECA-79 sulfated glycans (Figure 2, top panels), whereas MUC1 expression in perihilar-type ICC was primarily diffuse and cytoplasmic (Figure 2, third row, right panel). The expression pattern of MUC1 in peripheral-type ICC varied; however, most carcinoma cells exhibited both apical membrane and diffuse cytoplasmic patterns (Figure 2, second row, right panel). HCC tissue was completely negative for MUC1 core proteins (Figure 2, bottom right panel). Overall, these findings suggest that expression of MECA-79 sulfated glycans at the apical membrane of cholangiocytes indicates their cholangiolar/ductular differentiation and, compared with MUC1 positivity, appears to be a more sensitive diagnostic marker of CoCC and, to a lesser extent, of peripheral-type ICC, both of which likely originate from small-sized intrahepatic bile ducts.

\section{MUC1 is a Scaffold Protein for MECA-79 Sulfated Glycans} Using double immunofluorescence staining, we confirmed apical membrane colocalization of MECA-79 sulfated glycans and MUC1 core proteins in CoCC (Figure 3), prompting us to ask whether MUC1 is a potential scaffold protein for MECA-79 sulfated glycans. To determine this possibility, we transiently transfected $\mathrm{CHO}$ cells stably expressing MECA-79 sulfated glycans and $\mathrm{CD} 34$, which serves as a scaffold protein for MECA-79 sulfated glycans, ${ }^{27}$ with cDNA encoding FLAGtagged MUC1. In these transfectants, CD34 glycoforms serve as an internal positive control for glycosylation with MECA-79 sulfated glycans. We then immunoprecipitated cell lysates with anti-FLAG antibody or control mouse IgG and conducted Western blot analysis of precipitates plus samples from control lysates that had not undergone immunoprecipitation. Lysates from cells transfected with FLAG-tagged MUC1 showed a single band migrating at $180 \mathrm{kDa}$, a size corresponding to FLAG-tagged MUC1, whereas mocktransfected cells did not (Figure 4, left panel). A comparable band was also seen in samples immunoprecipitated with antiFLAG monoclonal antibody but was absent in samples immunoprecipitated with control mouse IgG.

Using the same samples, we then conducted Western blot analysis with a MECA-79 monoclonal antibody. As shown in Figure 4 (right panel), whole-cell lysates from mocktransfected cells showed a single $100 \mathrm{kDa}$ band corresponding to CD34 decorated with MECA-79 sulfated glycans, whereas lysates from cells transfected with FLAG-tagged MUC1 exhibited an additional band migrating at $180 \mathrm{kDa}$, corresponding to FLAG-tagged MUC1 glycoforms (Figure 4, right panel). After immunoprecipitation with anti-FLAG antibody, the CD34 band was absent and only the band corresponding to FLAG-tagged MUC1 remained, whereas no bands were 

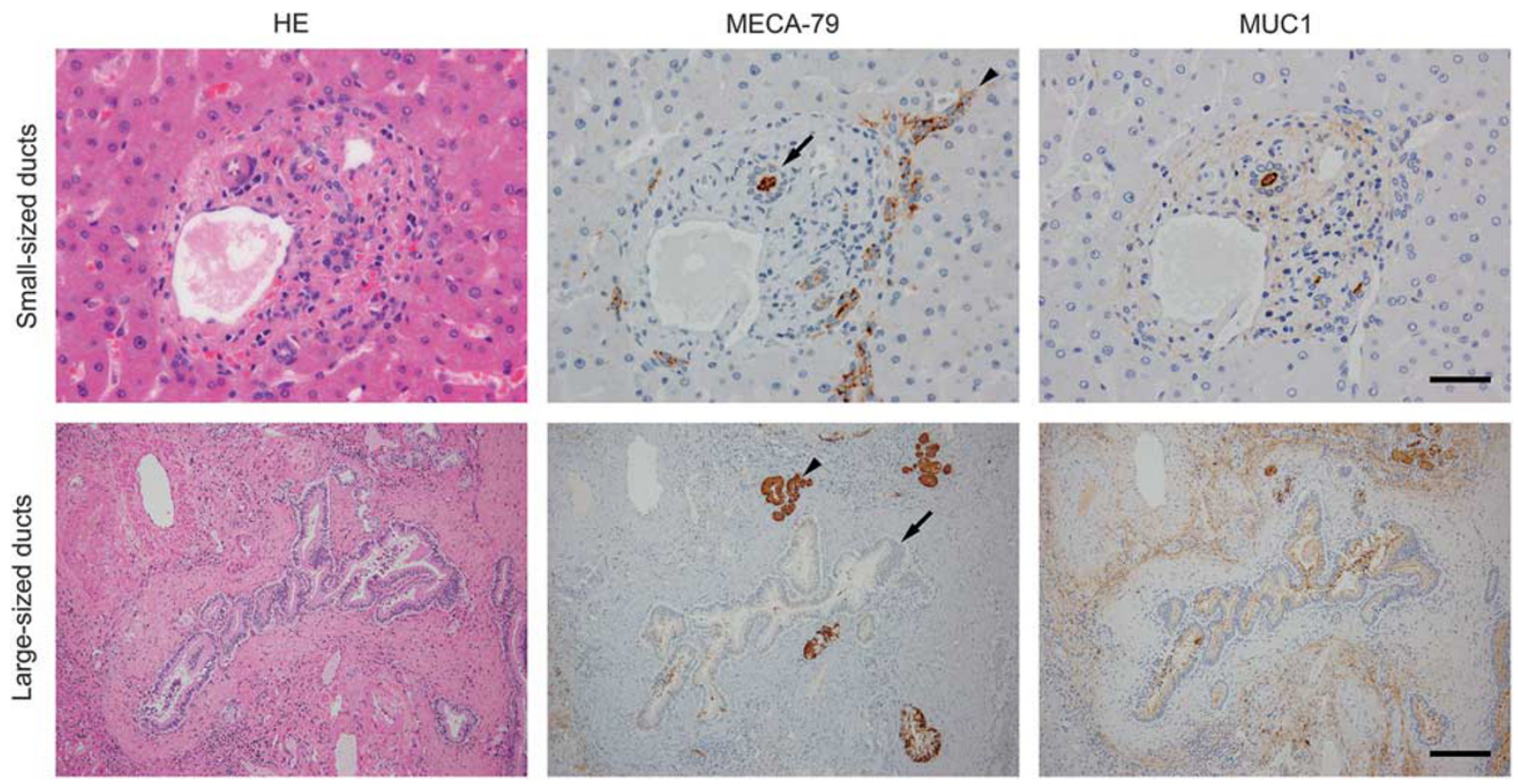

Figure 1 Expression of MECA-79 sulfated glycans and mucin 1 (MUC1) core proteins in small-sized (upper panels) and large-sized (lower panels) intrahepatic bile ducts. Normal human liver tissues were stained with hematoxylin and eosin (HE) and immunostained for MECA-79 and MUC1 core proteins. In small-sized intrahepatic bile ducts such as interlobular bile ducts (arrows), cholangioles/ductules and the canals of Hering (arrowheads), cholangiocytes are abundantly decorated with MECA-79 sulfated glycans, primarily in an apical membrane pattern (upper middle panel). However, in large-sized intrahepatic bile ducts, the largest ducts (arrows) are MECA-79 negative, and the smaller the ducts (including peribiliary accessory glands; arrowheads), the more MECA-79 positive they become, although staining becomes diffuse and cytoplasmic (lower middle panel). These expression patterns are comparable to those of MUC1 core proteins, except that (i) MECA-79 antibodies more widely recognize peripheral biliary tracts, including the canals of Hering and (ii) in large-sized intrahepatic bile ducts, MUC1 core proteins are expressed weakly in the cytoplasm of cholangiocytes (lower right panel). Tissues are counterstained with hematoxylin. Bar $=50 \mu \mathrm{m}$ (upper panels) and $200 \mu \mathrm{m}$ (lower panels).

evident in control IgG samples (Figure 4, right panel). These results indicate that MUC1 serves a scaffold protein for MECA-79 sulfated glycans.

\section{GlcNAc6ST-2 is Critical for Biosynthesis of MECA-79 Sulfated Glycans in Small-Sized Intrahepatic Bile Ducts and CoCC}

Among the five GlcNAc6ST family members cloned so far, ${ }^{46}$ GlcNAc6ST-1 (ref. 40) and GlcNAc6ST-2 (ref. 42) have been shown to cooperatively regulate sulfation of L-selectin ligand carbohydrates on HEVs. ${ }^{35,47}$ To determine which GlcNAc6ST is critical for sulfation in small-sized intrahepatic bile ducts and in CoCC, we conducted overexpression studies using the human ICC cell line SSP-25. These cells do not express MECA-79 sulfated glycans, as confirmed by RT-PCR analysis, which detected only minimal levels of GlcNAc6ST-1 and GlcNAc6ST-2 transcripts in these cells (Figure 5). We then transfected SSP-25 cells with either GlcNAc6ST-1 or GlcNAc6ST-2 expression vectors and observed that only cells overexpressing GlcNAc6ST-2 showed cell surface expression of MECA-79 sulfated glycans (Figure 6a), whereas those overexpressing GlcNAc6ST-1 did not (Figure 6b).

To confirm these findings in vivo, we examined liver tissues obtained from mice deficient in GlcNAc6ST-1 or doubly deficient in both GlcNAc6ST-1 and GlcNAc6ST-2 (DKO). As shown in Figure $6 \mathrm{c}$, in control wild-type mice, apical membrane expression of MECA-79 sulfated glycans was observed in $\sim 10 \%$ of interlobular bile ducts but not in the canals of Hering, whereas almost all small-sized intrahepatic bile ducts in human samples were MECA-79 positive (see Figure 1). Immunohistological findings seen in GlcNAc6ST-1-knockout mice were comparable to those seen in wild-type mice. By contrast, mice deficient in both GlcNAc6ST-1 and GlcNAc6ST-2 showed no expression of MECA-79 sulfated glycans, indicating that GlcNAc6ST-2 is the primary sulfotransferase catalyzing biosynthesis of MECA-79 sulfated glycans in interlobular bile ducts.

\section{DISCUSSION}

Here we show that MECA-79 sulfated glycans are constitutively expressed in apical membranes of cholangiocytes that comprise small-sized intrahepatic bile ducts, including interlobular bile ducts, cholangioles/ductules and the canals of Hering. Similar patterns of the MECA-79 immunolocalization observed in CoCC may reflect its cholangiolar/ductular differentiation. Thus, immunohistochemical positivity for MECA-79 glycoepitopes could represent an alternative means to diagnose CoCC. 

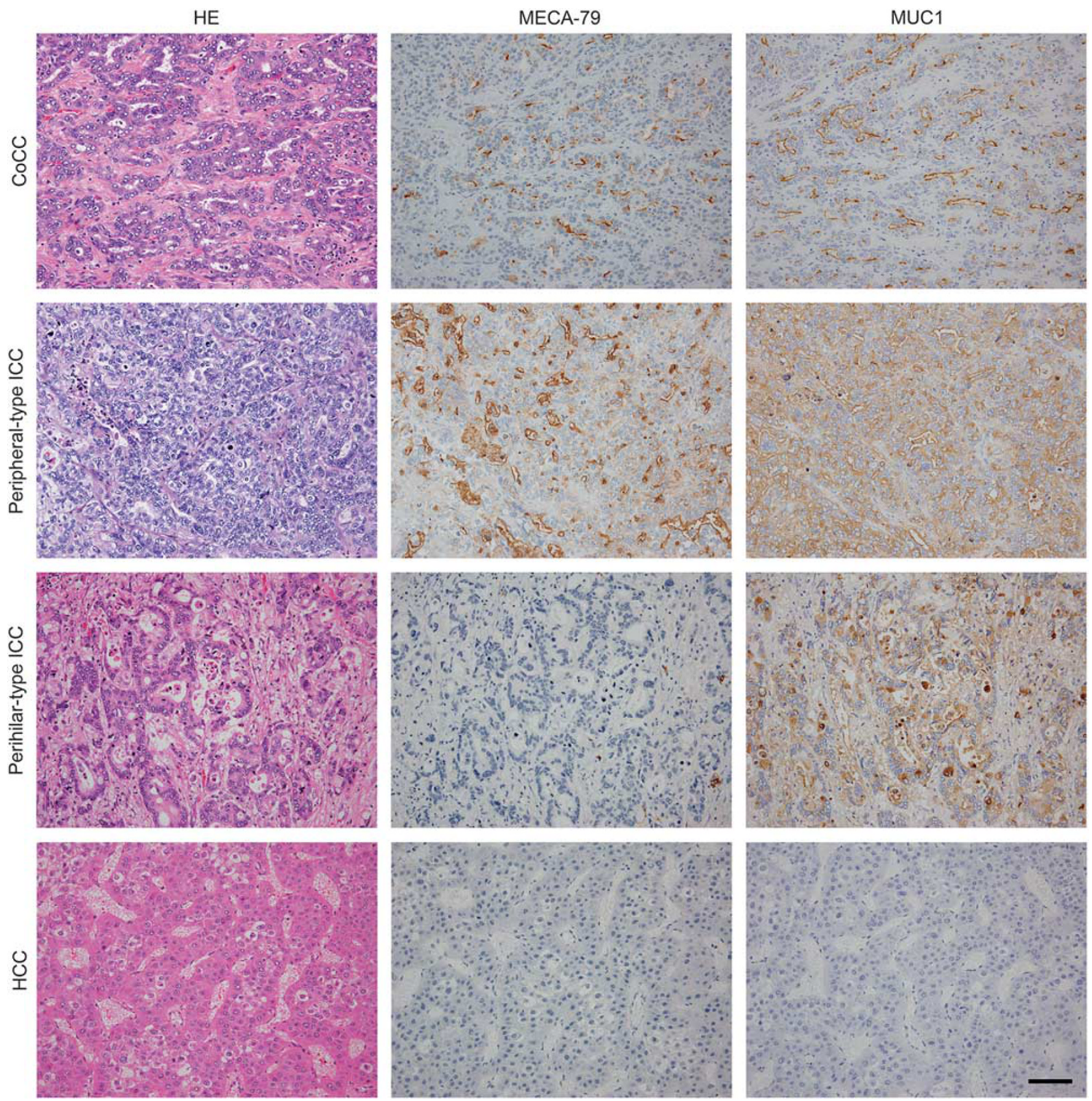

Figure 2 Expression of MECA-79 sulfated glycans and mucin 1 (MUC1) core proteins in cholangiolocellular carcinoma (CoCC) (top row), peripheral-type intrahepatic cholangiocarcinoma (ICC) (second row), perihilar-type ICC (third row) and hepatocellular carcinoma (HCC) (bottom row). Serial sections were stained with hematoxylin and eosin (HE) and immunostained for MECA-79 and MUC1 core proteins. MECA-79 sulfated glycans are preferentially expressed along the luminal surface of canalicular structures formed in CoCC and peripheral-type ICC, an expression pattern comparable to MUC1 core proteins. Tissues are counterstained with hematoxylin. Bar $=100 \mu \mathrm{m}$.

Although MECA-79 monoclonal antibody more selectively recognizes peripheral small-sized intrahepatic bile ducts, centrally located large-sized biliary tract epithelia and their accessory mucus-secreting glands in normal liver tissues are almost devoid of apical membrane expression of MECA-79 sulfated glycans. Maeno et $\mathrm{al}^{16}$ reported site-dependent, differential localization patterns of MUC1 core proteins. Accordingly, they found that central biliary ducts wider than $40 \mu \mathrm{m}$ lacked apical membrane MUC1 expression, whereas interlobular bile ducts and their peripheral ductular cells exhibited an apical membrane staining pattern. These results are in agreement with those reported here; however, here, we show that MECA-79 sulfated glycans are a more sensitive marker of components of the peripherally distributed biliary ductular system, including the canals of Hering. 
MECA-79

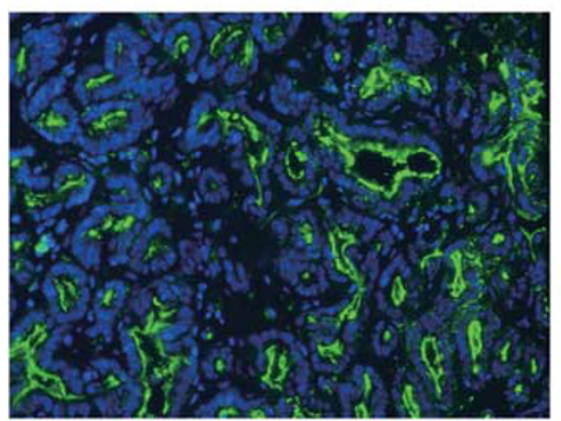

MUC1

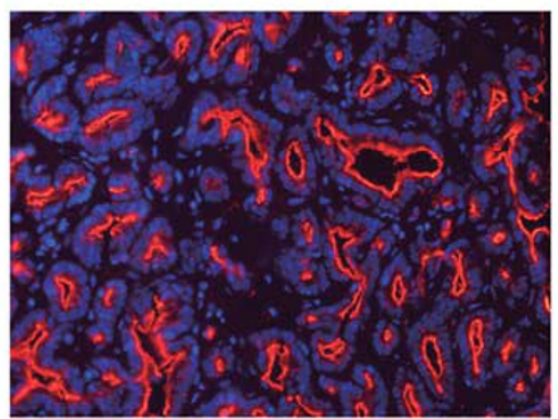

Merged

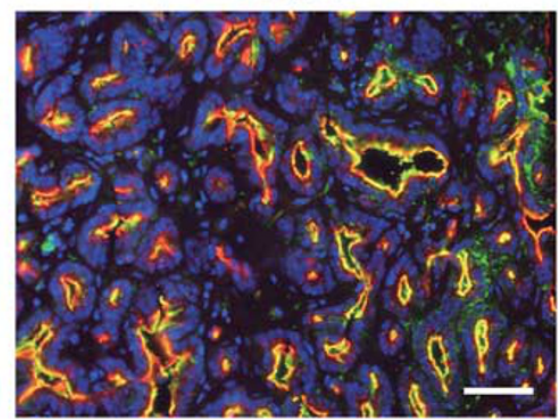

Figure 3 Double immunofluorescence staining of cholangiolocellular carcinoma (CoCC) for MECA-79 sulfated glycans (green) and mucin 1 (MUC1) core proteins (red). Merged panels indicate colocalization. Samples are stained with 4',6-diamidino-2-phenylindole (DAPI) to mark nuclei. Bar $=100 \mu$ m.

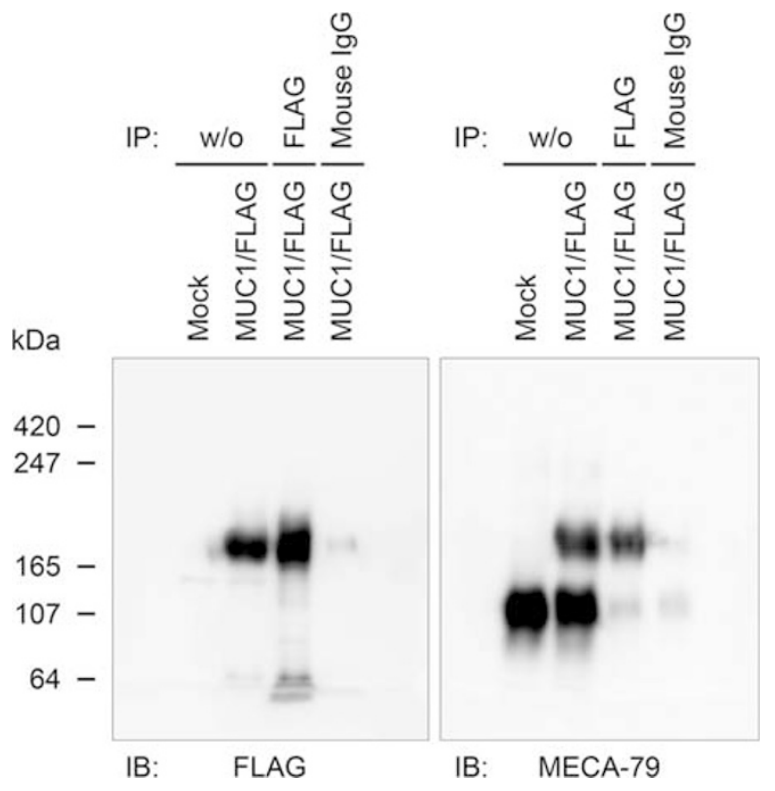

Figure 4 Mucin 1 (MUC1) proteins are decorated with MECA-79 sulfated glycans. Chinese hamster ovary (CHO) cells expressing CD34 decorated with MECA-79 sulfated glycans were transiently transfected with pcDNA3.1-MUC1/FLAG (MUC1/FLAG) or pcDNA3.1 (mock). Whole-cell lysates immunoprecipitated (IP'd) with anti-FLAG M2 antibody or control non-immune mouse immunoglobulin $\mathrm{G}(\mathrm{lgG})$, as well as those without (w/o) immunoprecipitation, were analyzed by Western blotting (IB) with anti-FLAG M2 (left panel) or MECA-79 (right panel) monoclonal antibodies. Bands migrating at $180 \mathrm{kDa}$ correspond to FLAG epitopetagged MUC1; those migrating at $100 \mathrm{kDa}$ correspond to CD34. Numbers at left indicate molecular weight $(\mathrm{kDa})$.

In the present study, we used Western blot analysis with MECA-79 antibody of immunoprecipitated recombinant FLAG-tagged MUC1 to demonstrate that MUC1 can serve as a scaffold protein for MECA-79 sulfated glycans. We performed this analysis in MECA-79-positive $\mathrm{CHO}$ cells that had been transiently transfected with a MUC1 expression vector as a model of CoCC cells, as no CoCC cell lines are currently available. Future experiments using either CoCC carcinoma cells or cell lines derived from CoCC should be
Table 1 Apical membrane expression of MECA-79 sulfated glycans in primary liver tumors

\begin{tabular}{lcccc}
\hline & \multicolumn{3}{c}{ ICC $(n=71)$} & $\begin{array}{c}\text { HCC } \\
(n=25)\end{array}$ \\
\cline { 2 - 3 } & $\begin{array}{c}\text { Peripheral type } \\
\text { (in a broad sense) }(n=46)\end{array}$ & $\begin{array}{c}\text { Perihilar type } \\
(n=25)\end{array}$ & \\
\cline { 2 - 3 } & $\begin{array}{c}\text { CoCC } \\
(n=20)\end{array}$ & $\begin{array}{c}\text { Peripheral type } \\
(n=26)\end{array}$ & & \\
\hline MECA-79 (+) & $18(90 \%)$ & $12(46.2 \%)$ & $0(0 \%)$ & $0(0 \%)$ \\
MECA-79 (-) & $2(10 \%)$ & $14(53.8 \%)$ & $25(100 \%)$ & $25(100 \%)$ \\
\hline
\end{tabular}

Abbreviations: CoCC, cholangiolocellular carcinoma; HCC, hepatocellular carcinoma; ICC, intrahepatic cholangiocarcinoma.

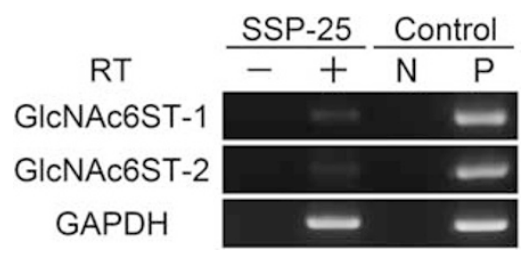

Figure 5 Reverse transcription-PCR (RT-PCR) analysis of transcripts encoding GlcNAc6ST-1 and GlcNAc6ST-2 in SSP-25 human intrahepatic cholangiocarcinoma (ICC) cells. RNA samples were treated with (+) or without (-) RT. Distilled water (N) or plasmid harboring target CDNA (P), respectively, served as negative or positive controls. SSP-25 cells show minimal expression of transcripts encoding either sulfotransferase. GAPDH (glyceraldehyde-3-phosphate dehydrogenase) serves as a loading control.

performed to confirm these findings and define molecular mechanisms governing apical membrane expression of sulfated glycans in cholangiocytes with cholangiolar/ductular differentiation.

Sasaki et $a l^{48}$ previously reported that MUC6 core proteins are expressed on the luminal surface of small-sized intrahepatic bile ducts, particularly proliferating bile ductules seen in chronic viral hepatitis, as well as on canalicular structures formed in various types of ICC, including combined 
a

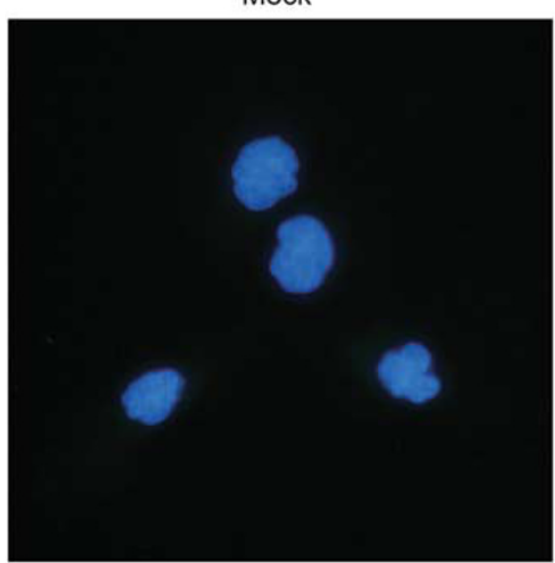

b

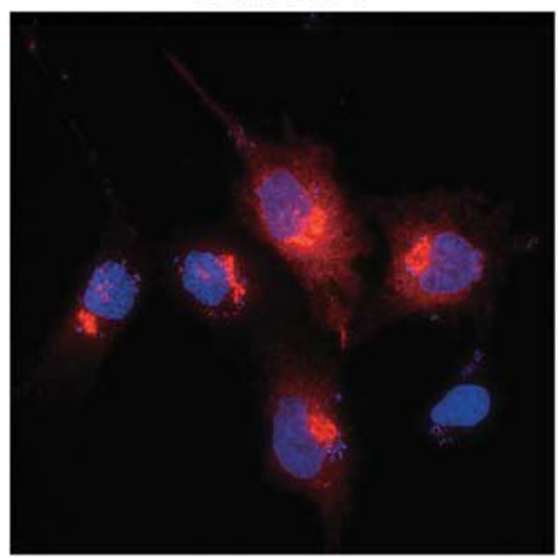

C

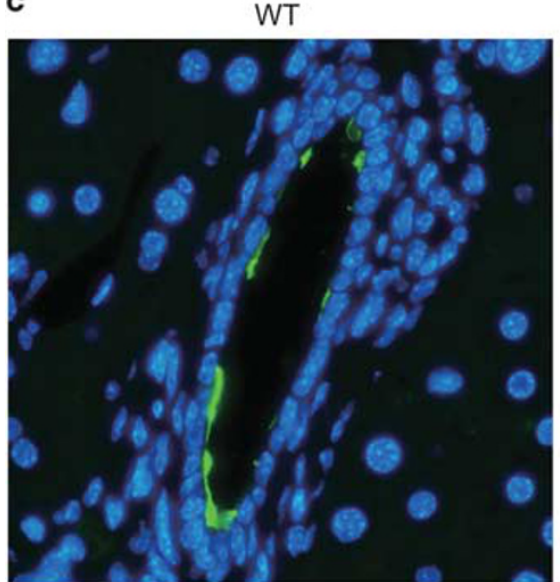

GlcNAc6ST-1

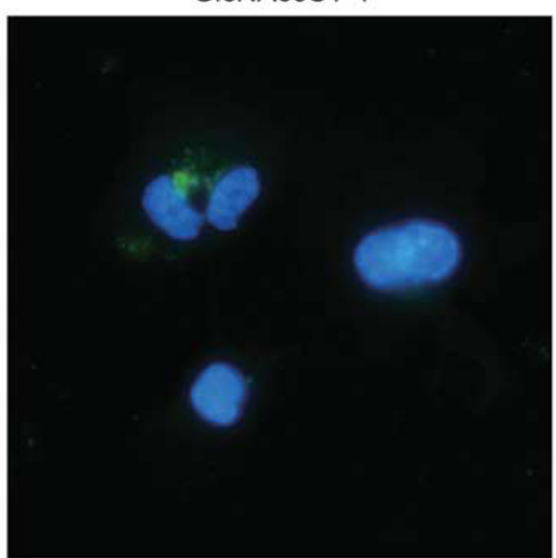

MECA-79

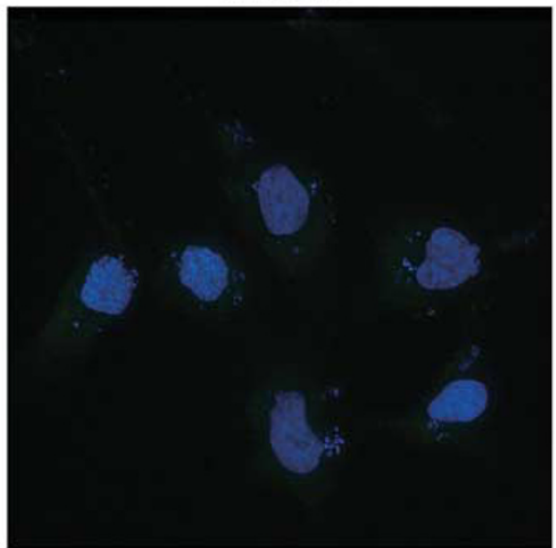

GIcNAc6ST-1 KO

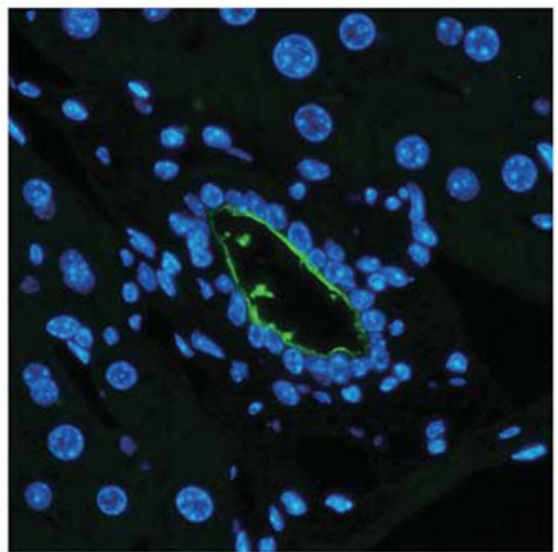

GlcNAc6ST-2

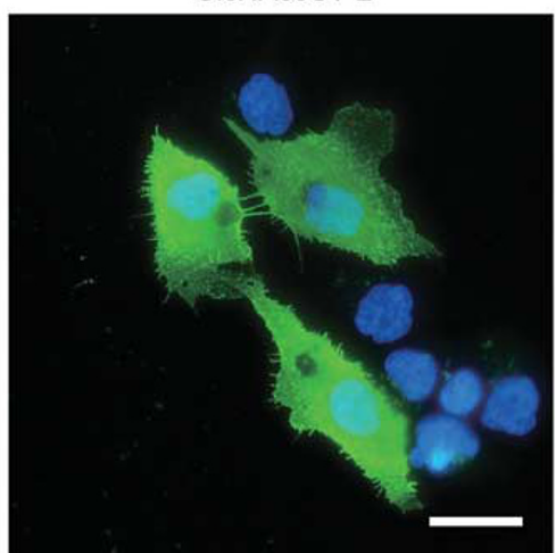

Merged

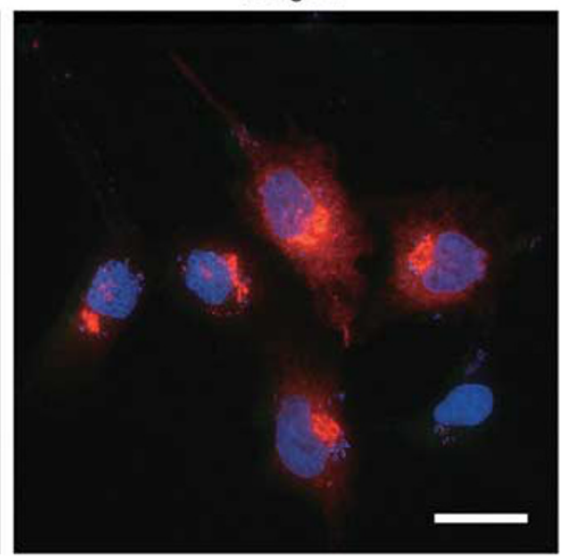

GlcNAc6ST-1/2 DKO

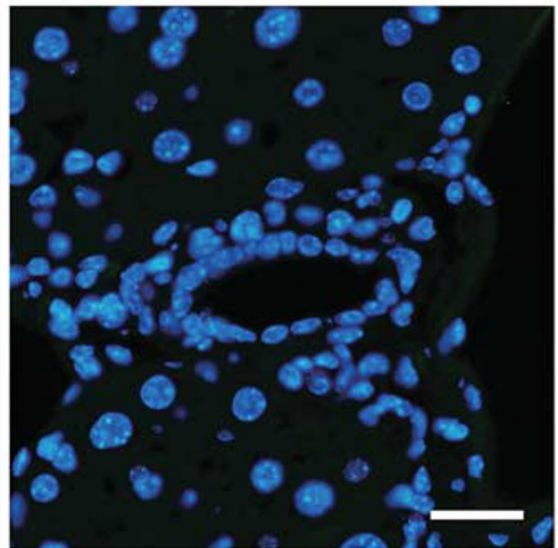

Figure 6 (a) Immunofluorescence staining (green) for MECA-79 sulfated glycans in SSP-25 cells transiently transfected with pcDNA1.1 (mock), pcDNA1.1GlcNAc6ST-1, or pcDNA1.1-GlcNAc6ST-2. (b) SSP-25 cells transiently transfected with GlcNAc6ST-1 cDNA were subjected to double immunofluorescence staining for GlcNAc6ST-1 proteins (red) and MECA-79 sulfated glycans (green). Despite GlcNAc6ST-1 misexpression, MECA-79 sulfated glycans are not detectable. (c) Immunofluorescence staining (green) for MECA-79 sulfated glycans in liver tissue sections obtained from wild-type mice (WT), mice deficient in GlcNAc6ST-1 (GlcNAcST-1 KO), and mice deficient in both GlcNAc6ST-1 and GlcNAc6ST-2 (GlcNAc6ST-1/2 DKO). Samples are also stained with 4',6-diamidino-2-phenylindole (DAPI) to indicate nuclei. Bar $=50 \mu \mathrm{m}$. 
hepatocellular-cholangiocarcinoma. ${ }^{49}$ Such MUC6 immunolocalization resembles that of $\mathrm{MUC1}$, as previously reported by the same authors ${ }^{15,50}$ and confirmed here. Thus, in addition to MUC1, MUC6 may also serve as a scaffold protein for MECA-79 sulfated glycans in cholangiocytes in small-sized intrahepatic bile ducts or in CoCC cells.

Here, we also demonstrate that GlcNAc6ST-2 is likely the principal sulfotransferase catalyzing biosynthesis of MECA-79 sulfated glycans in CoCC, as transfection of the human ICC cell line SSP-25 (which is negative for MECA-79 sulfated glycans) with a construct encoding GlcNAc6ST-2, but not GlcNAc6ST-1, promoted membrane expression of MECA-79 sulfated glycans. Moreover, using Northern blot analysis, Bistrup et $a l^{42}$ clearly demonstrated that GlcNAc6ST-2 transcripts were present not only in lymph nodes but also in liver and pancreas, where ductal epithelial cells express MECA-79 sulfated glycans, as we have shown here and previously. ${ }^{33}$ In support of these findings in humans, we also report here that MECA-79 expression in interlobular bile ducts, which is seen in wild-type and GlcNAc6ST-1-knockout mice, is absent in mice doubly deficient in GlcNAc6ST-1 and GlcNAc6ST-2. Overall, these findings strongly suggest that GlcNAc6ST-2 has a critical role in the biosynthesis of MECA-79 sulfated glycans in small-sized intrahepatic bile ducts and in CoCC.

In an unanticipated finding, we observed that in wild-type or GlcNAc6ST-1-knockout mice, MECA-79 sulfated glycan expression was seen in a fraction $(\sim 10 \%)$ of interlobular bile ducts but not in cholangioles/ductules and the canals of Hering, whereas in human tissues, most small-sized intrahepatic bile ducts were MECA-79 positive. This species difference may be due to differences in expression levels of core 1 extending $\beta 1,3-N$-acetylglucosaminyltransferase, also called core 1 extension enzyme, an enzyme required for MECA-79 glycoepitope biosynthesis. ${ }^{32,51}$ Alternatively, expression levels of GlcNAc6ST-2 and/or MUC1 core proteins may be lower in the murine biliary system than in the human system.

In malignant tumors, GlcNAc6ST-2-mediated expression of MECA-79 sulfated glycans is not restricted to liver, pancreas or lymph nodes: Kanoh et $a l^{52}$ report that GlcNAc6ST-2 is expressed in subtypes of ovarian carcinoma such as mucinous, clear cell, and serous papillary carcinomas. Moreover, Okayama et $a l^{53}$ reported that MECA-79 sulfated glycans were aberrantly expressed in roughly a quarter of gastric adenocarcinoma cases. Recently, we also reported that $\sim 20 \%$ of bladder urothelial carcinoma cases express MECA-79 sulfated glycans. ${ }^{38}$ In both ours ${ }^{38}$ and the Okayama et $a^{53}$ study, GlcNAc6ST-2 was identified as the sulfotransferase responsible for biosynthesis of MECA-79 sulfated glycans expressed on carcinoma cells. These findings indicate that aberrant GlcNAc6ST-2 expression has a major role in inappropriate biosynthesis of MECA-79 sulfated glycans in neoplastic cells in organs other than liver, pancreas and lymph nodes.
In conclusion, we show here that preferential expression of GlcNAc6ST-2-mediated MECA-79 sulfated glycans at apical membranes of cholangiocytes is indicative of cholangiolar/ ductular differentiation. We propose that MECA-79 sulfated glycans could serve as a useful marker of CoCC.

Supplementary Information accompanies the paper on the Laboratory Investigation website (http://www.laboratoryinvestigation.org)

\section{ACKNOWLEDGMENTS}

We thank Hisataka Kato and Noriko Maruta for technical assistance, Dr Ayumi Ohya for useful discussion, Dr Yasuhiro Maruyama for assisting with patient follow-up, and Dr Elise Lamar for critical reading of the manuscript. This work was supported by Grant-in-Aid for Young Scientists 15K19049 (to HH) and, in part, by Grant-in-Aid for Scientific Research $15 \mathrm{~K} 08265$ (to KU) and $15 \mathrm{~K} 08343$ (to MK) from the Japan Society for the Promotion of Science. This work was also supported by Grant Award from the Hokuto Foundation for Bioscience (to MK).

\section{DISCLOSURE/CONFLICT OF INTEREST}

The authors declare no conflict of interest.

1. $\mathrm{Yu} \mathrm{TH}, \mathrm{Yuan} \mathrm{RH}, \mathrm{Chen} \mathrm{YL}$, et al. Viral hepatitis is associated with intrahepatic cholangiocarcinoma with cholangiolar differentiation and $\mathrm{N}$-cadherin expression. Mod Pathol 2011;24:810-819.

2. Liau JY, Tsai JH, Yuan $\mathrm{RH}$, et al. Morphological subclassification of intrahepatic cholangiocarcinoma: etiological, clinicopathological, and molecular features. Mod Pathol 2014;27:1163-1173.

3. Kozaka K, Sasaki M, Fujii K, et al. A subgroup of intrahepatic cholangiocarcinoma with an infiltrating replacement growth pattern and a resemblance to reactive proliferating bile ductules: 'bile ductular carcinoma'. Histopathology 2007;51:390-400.

4. Aishima $S$, Kuroda $Y$, Nishihara $Y$, et al. Proposal of progression model for intrahepatic cholangiocarcinoma: clinicopathologic differences between hilar type and peripheral type. Am J Surg Pathol 2007;31: 1059-1067.

5. Komuta M, Govaere O, Vandecaveye V, et al. Histological diversity in cholangiocellular carcinoma reflects the different cholangiocyte phenotypes. Hepatology 2012;55:1876-1888.

6. Aishima S, Oda Y. Pathogenesis and classification of intrahepatic cholangiocarcinoma: different characters of perihilar large duct type versus peripheral small duct type. J Hepatobiliary Pancreat Sci 2015;22: 94-100.

7. Gandou C, Harada K, Sato Y, et al. Hilar cholangiocarcinoma and pancreatic ductal adenocarcinoma share similar histopathologies, immunophenotypes, and development-related molecules. Hum Pathol 2013;44:811-821.

8. Roskams TA, Theise ND, Balabaud C, et al. Nomenclature of the finer branches of the biliary tree: canals, ductules, and ductular reactions in human livers. Hepatology 2004;39:1739-1745.

9. Theise ND, Nakashima O, Park YN, et al. Combined hepatocellularcholangiocarcinoma. In: Bosman FT, Carneiro F, Hruban RH, et al. (eds). WHO Classification of Tumors of the Digestive System, 4th edn. International Agency for Research on Cancer: Lyon, France, 2010, pp 225-227.

10. Steiner PE, Higginson J. Cholangiolocellular carcinoma of the liver. Cancer 1959;12:753-759.

11. Komuta M, Spee B, Vander Borght S, et al. Clinicopathological study on cholangiolocellular carcinoma suggesting hepatic progenitor cell origin. Hepatology 2008;47:1544-1556.

12. Theise ND, Saxena R, Portmann BC, et al. The canals of Hering and hepatic stem cells in humans. Hepatology 1999;30:1425-1433.

13. Roskams T. Liver stem cells and their implication in hepatocellular and cholangiocarcinoma. Oncogene 2006;25:3818-3822.

14. Gütgemann I, Haas S, Berg JP, et al. CD56 expression aids in the differential diagnosis of cholangiocarcinomas and benign cholangiocellular lesions. Virchows Arch 2006;448:407-411. 
15. Sasaki $M$, Nakanuma Y. Expression of mucin core protein of mammary type in primary liver cancer. Hepatology 1994;20:1192-1197.

16. Maeno S, Kondo F, Sano K, et al. Morphometric and immunohistochemical study of cholangiolocellular carcinoma: comparison with non-neoplastic cholangiole, interlobular duct and septal duct. J Hepatobiliary Pancreat Sci 2012;19:289-296.

17. Burdick MD, Harris A, Reid CJ, et al. Oligosaccharides expressed on MUC1 produced by pancreatic and colon tumor cell lines. J Biol Chem 1997;272:24198-24202.

18. Hanisch FG, Müller S. MUC1: the polymorphic appearance of a human mucin. Glycobiology 2000;10:439-449.

19. Ligtenberg MJ, Kruijshaar L, Buijs F, et al. Cell-associated episialin is a complex containing two proteins derived from a common precursor. J Biol Chem 1992;267:6171-6177.

20. Swanson BJ, McDermott KM, Singh PK, et al. MUC1 is a counterreceptor for myelin-associated glycoprotein (Siglec-4a) and their interaction contributes to adhesion in pancreatic cancer perineural invasion. Cancer Res 2007;67:10222-10229.

21. Gendler SJ, Lancaster CA, Taylor-Papadimitriou J, et al. Molecular cloning and expression of human tumor-associated polymorphic epithelial mucin. J Biol Chem 1990;265:15286-15293.

22. Hanski C, Drechsler K, Hanisch FG, et al. Altered glycosylation of the MUC-1 protein core contributes to the colon carcinoma-associated increase of mucin-bound sialyl-Lewis X expression. Cancer Res 1993;53: 4082-4088.

23. Butcher EC, Picker LJ. Lymphocyte homing and homeostasis. Science 1996;272:60-66

24. Kobayashi M, Mitoma J, Nakamura N, et al. Induction of peripheral lymph node addressin in human gastric mucosa infected by Helicobacter pylori. Proc Natl Acad Sci USA 2004;101:17807-17812.

25. Okamura $T$, Sakai $Y$, Hoshino $H$, et al. Superficially located enlarged lymphoid follicles characterise nodular gastritis. Pathology 2015;47: 38-44.

26. Suzawa $K$, Kobayashi $M$, Sakai $Y$, et al. Preferential induction of peripheral lymph node addressin on high endothelial venule-like vessels in the active phase of ulcerative colitis. Am J Gastroenterol 2007:102:1499-1509.

27. Kobayashi M, Hoshino $H$, Masumoto J, et al. GlcNAc6ST-1-mediated decoration of MAdCAM-1 protein with L-selectin ligand carbohydrates directs disease activity of ulcerative colitis. Inflamm Bowel Dis 2009;15: 697-706.

28. Hemmerich S, Butcher EC, Rosen SD. Sulfation-dependent recognition of high endothelial venules (HEV)-ligands by L-selectin and MECA 79, and adhesion-blocking monoclonal antibody. J Exp Med 1994;180: 2219-2226.

29. Mitsuoka C, Sawada-Kasugai M, Ando-Furui K, et al. Identification of a major carbohydrate capping group of the L-selectin ligand on high endothelial venules in human lymph nodes as 6-sulfo sialyl Lewis X. J Biol Chem 1998;273:11225-11233.

30. Rosen SD. Ligands for L-selectin: homing, inflammation, and beyond. Annu Rev Immunol 2004;22:129-156.

31. Streeter PR, Rouse BT, Butcher EC. Immunohistologic and functional characterization of a vascular addressin involved in lymphocyte homing into peripheral lymph nodes. J Cell Biol 1988;107: 1853-1862.

32. Yeh JC, Hiraoka N, Petryniak B, et al. Novel sulfated lymphocyte homing receptors and their control by a core 1 extension $\beta 1,3-N$ acetylglucosaminyltransferase. Cell 2001;105:957-969.

33. Maruyama $M$, Kobayashi $M$, Sakai $Y$, et al. Periductal induction of high endothelial venule-like vessels in type 1 autoimmune pancreatitis. Pancreas 2013:42:53-59.

34. Uchimura K, Kadomatsu K, El-Fasakhany FM, et al. N-acetylglucosamine 6-O-sulfotransferase-1 regulates expression of L-selectin ligands and lymphocyte homing. J Biol Chem 2004;279:35001-35008.
35. Uchimura K, Gauguet JM, Singer MS, et al. A major class of L-selectin ligands is eliminated in mice deficient in two sulfotransferases expressed in high endothelial venules. Nat Immunol 2005;6: $1105-1113$.

36. Price MR, Rye PD, Petrakou E, et al. Summary report on the ISOBM TD-4 Workshop: analysis of 56 monoclonal antibodies against the MUC1 mucin. San Diego, Calif., November 17-23, 1996. Tumour Biol 1998;19 (Suppl 1):1-20.

37. Kobayashi M, Nakayama J. Immunohistochemical analysis of carbohydrate antigens in chronic inflammatory gastrointestinal diseases. Methods Enzymol 2010;479:271-289.

38. Taga $\mathrm{M}$, Hoshino $\mathrm{H}$, Low $\mathrm{S}$, et al. A potential role for 6-sulfo sialyl Lewis $X$ in metastasis of bladder urothelial carcinoma. Urol Oncol 2015;33: 496.e1-469.e9.

39. Kobayashi M, Mitoma J, Hoshino $\mathrm{H}$, et al. Prominent expression of sialy Lewis X-capped core 2-branched O-glycans on high endothelial venule-like vessels in gastric MALT lymphoma. J Pathol 2011;224: 67-77.

40. Uchimura $\mathrm{K}$, Muramatsu $\mathrm{H}$, Kaname $\mathrm{T}$, et al. Human $\mathrm{N}$-acetylglucosamine-6-O-sulfotransferase involved in the biosynthesis of 6-sulfo sialyl Lewis X: molecular cloning, chromosomal mapping, and expression in various organs and tumor cells. J Biochem 1998;124: 670-678.

41. Fujiwara $M$, Kobayashi $M$, Hoshino $H$, et al. Expression of long-form $\mathrm{N}$-acetylglucosamine-6-O-sulfotransferase 1 in human high endothelial venules. J Histochem Cytochem 2012;60:397-407.

42. Bistrup A, Bhakta S, Lee JK, et al. Sulfotransferases of two specificities function in the reconstitution of high endothelial cell ligands for L-selectin. J Cell Biol 1999;145:899-910.

43. Ho SN, Hunt HD, Horton RM, et al. Site-directed mutagenesis by overlap extension using the polymerase chain reaction. Gene 1989;77: 51-59.

44. Hu G, Fearon ER. Siah-1 N-terminal RING domain is required for proteolysis function, and C-terminal sequences regulate oligomerization and binding to target proteins. Mol Cell Biol 1999;19:724-732.

45. Hoshino $\mathrm{H}$, Kobayashi $\mathrm{M}$, Mitoma J, et al. An integrin $a 4 \beta 7 \cdot \mathrm{lgG}$ heterodimeric chimera binds to MAdCAM-1 on high endothelial venules in gut-associated lymphoid tissue. J Histochem Cytochem 2011:59:572-583.

46. Uchimura K, Rosen SD. Sulfated L-selectin ligands as a therapeutic target in chronic inflammation. Trends Immunol 2006;27:559-565.

47. Kawashima H, Petryniak B, Hiraoka N, et al. N-acetylglucosamine-6-Osulfotransferases 1 and 2 cooperatively control lymphocyte homing through L-selectin ligand biosynthesis in high endothelial venules. Nat Immunol 2005;6:1096-1104.

48. Sasaki M, Nakanuma $Y$, Ho SB, et al. Increased MUC6 apomucin expression is a characteristic of reactive biliary epithelium in chronic viral hepatitis. J Pathol 1998;185:191-198.

49. Sasaki $M$, Nakanuma $Y, H o ~ S B$, et al. Cholangiocarcinomas arising in cirrhosis and combined hepatocellular-cholangiocellular carcinomas share apomucin profiles. Am J Clin Pathol 1998;109:302-308.

50. Sasaki M, Nakanuma Y. Frequent expression of MUC1 apomucin on biliary epithelial cells of damaged small bile ducts in primary biliary cirrhosis and chronic viral hepatitis: an immunohistochemical study. Hepatology 1996;23:1313-1317.

51. Mitoma J, Bao X, Petryanik B, et al. Critical functions of $\mathrm{N}$-glycans in L-selectin-mediated lymphocyte homing and recruitment. Nat Immunol 2007;8:409-418.

52. Kanoh $\mathrm{A}$, Seko $\mathrm{A}$, Ideo $\mathrm{H}$, et al. Ectopic expression of $\mathrm{N}$-acetylglucosamine 6-O-sulfotransferase 2 in chemotherapy-resistant ovarian adenocarcinomas. Glycoconj J 2006;23:453-460.

53. Okayama H, Kumamoto K, Saitou K, et al. Ectopic expression of MECA-79 as a novel prognostic indicator in gastric cancer. Cancer Sci 2011;102:1088-1094. 